\title{
Expansive Phenotypic Landscape of Botrytis cinerea Shows Differential Contribution of Genetic Diversity and Plasticity
}

\author{
Jason A. Corwin, ${ }^{1}$ Anushriya Subedy, ${ }^{1}$ Robert Eshbaugh, ${ }^{1}$ and Daniel J. Kliebenstein ${ }^{1,2}$ \\ ${ }^{1}$ Department of Plant Sciences, University of California, Davis, One Shields Avenue, Davis, CA 95616, U.S.A.; and ${ }^{2}$ DynaMo \\ Center of Excellence, University of Copenhagen, Thorvaldsensvej 40, DK-1871, Frederiksberg C, Denmark
}

Submitted 2 September 2015. Accepted 8 January 2016.

\begin{abstract}
The modern evolutionary synthesis suggests that both environmental variation and genetic diversity are critical determinants of pathogen success. However, the relative contribution of these two sources of variation is not routinely measured. To estimate the relative contribution of plasticity and genetic diversity for virulence-associated phenotypes in a generalist plant pathogen, we grew a population of 15 isolates of Botrytis cinerea from throughout the world, under a variety of in vitro and in planta conditions. Under in planta conditions, phenotypic differences between the isolates were determined by the combination of genotypic variation within the pathogen and environmental variation. In contrast, phenotypic differences between the isolates under in vitro conditions were predominantly determined by genetic variation in the pathogen. Using a correlation network approach, we link the phenotypic variation under in vitro experimental conditions to phenotypic variation during plant infection. This study indicates that there is a high level of phenotypic variation within $B$. cinerea that is controlled by a mixture of genetic variation, environment, and genotype $\times$ environment. This argues that future experiments into the pathogenicity of $B$. cinerea must account for the genetic and environmental variation within the pathogen to better sample the potential phenotypic space of the pathogen.
\end{abstract}

Sessile organisms, including many plant pathogens, must be able to cope or adapt to changes and heterogeneities in their local environment. These heterogeneities include a variety of abiotic stresses such as temperature and water availability but, for plant pathogens, can also include diverse biotic stresses imposed by the host, such as passive physical barriers, pre- or post-immune responses, and chemical defenses across an array of potential hosts. These environmental changes and associated selective pressures can influence how the pathogen evolves an appropriate phenotypic response and influences the level of standing genetic variation within the pathogen. Evolutionary pressures from changing environments can lead to the development of plasticity, in which individual genotypes respond to their environment using signal transduction pathways to detect changes in their local environment and respond by modifying

Corresponding author: D. J. Kliebenstein;

E-mail: kliebenstein@ucdavis.edu

*The e-Xtra logo stands for "electronic extra" and indicates that seven supplementary figures and five supplementary tables are published online.

(c) 2016 The American Phytopathological Society their morphology, physiology, and biochemistry (Agrawal 2001; Bradshaw 1965; Schlichting 1986). This allows a pathogen, especially generalists, to counteract diverse host immune responses and develop in a manner that optimizes its growth and virulence in planta across a range of stress types and severities. For example, a fungal pathogen can alter its growth rate in response to the level of host resistance (Rowe et al. 2010).

In addition to signal transduction-mediated responses to diverse resistance mechanisms, a pathogen could consist of a large population of individuals that contain many distinct allelic combinations that convey differential virulence on a particular host. The host's innate immune responses can then impose selection on this pathogen population, leading to enhanced fitness of pathogen individuals that have more virulent allelic combinations (Barrett and Schluter 2008; McDonald and Linde 2002). If individuals that infect different hosts then mate and reshuffle their genetic diversity via meiotic or parasexual processes, they could potentially regenerate the population's initial mix of diversity while alleviating the linkage-drag effects of any selective or demographic bottlenecks in previous generations. This dynamic process of heterogeneous, fluctuating selection and reshuffling would allow genetic diversity to be maintained in the pathogen (Kerwin et al. 2015; Turelli and Barton 2004). Given that generalist fungal pathogens with a broad host range are likely to encounter heterogeneous hosts and corresponding defenses from generation to generation, it is possible that fluctuating selection from these diverse host plant defense mechanisms may act to maintain phenotypic diversity within a generalist pathogen. While there have been innumerable studies on the environmental or genetic variation on individual traits or genes or markers, there have been very few surveys that investigate the combination of genotypic and environmental variation on a broad scope of trait diversity in a collection of generalist pathogens to assess the relative contribution of plasticity versus genetic variation in virulence-related traits.

It is important to understand the relative contributions of plasticity and standing genetic variation for virulence-associated traits so that we can design appropriate experiments for exploring their functional underpinnings. Within this paper, we define plasticity as the ability of a single genotype of an organism to detect changes in their environment and use signal transduction pathways to alter their phenotype in response to environmental change (Forsman 2014; Price et al. 2003). For example, Arabidopsis thaliana senses changes in nutrient stress and light intensity and, then, utilizes regulatory machinery to alter their phenotype in response. This process is largely driven by environmental inputs rather than natural genetic difference among individuals (Pigliucci and Byrd 1998; Pigliucci and 
Kolodynska 2001; Pigliucci and Schlichting 1996). In addition, the soil fungus and facultative epiphyte Aureobasidium pullulans demonstrates a variety of genetically independent morphological traits in response to differences in nutrient source and temperature (Slepecky and Starmer 2009). This variation can be easily assessed, within a population of individuals, utilizing a linear modeling approach such as an analysis of variance (Whitman and Agrawal 2009). The identification of plasticity requires measuring the trait of individual genotypes across a diversity of environments. These environments could include different in vitro growth media or even different plant genotypes that vary in their innate immune response (e.g., reactive oxygen species levels, defense compounds), which would cause the pathogen to experience different environments in planta. In contrast, identifying pathogen traits whose phenotypic variation is largely linked to standing genetic diversity requires measuring variation of the phenotype across many different pathogen genotypes to elucidate the differences between genotypes. It is possible that genetically polymorphic traits in a generalist pathogen may be involved in optimizing virulence across a range of unpredictable host species. Thus, by measuring the phenotypic response of a genetically diverse collection of individuals across a broad set of environments, we can use a linear modeling approach to dissect each trait into its plastic (variation within a genotype across environments) and genetic (variation across genotypes) components. This can then be used to assess if there are any differences in how traits partition between plastic and genetic variance.

To better partition plasticity versus genetic diversity, it would be optimal to use a pathogen that has a large amount of standing genetic diversity and demonstrates quantitative virulence across a broad host range to measure the differential contribution of phenotypic plasticity and genetic diversity. Therefore, we chose to investigate selected virulence-associated phenotypes within the model plant pathogen Botrytis cinerea. B. cinerea is a necrotrophic fungal plant pathogen with an extremely broad host range and is responsible for postharvest crop loss across the globe (Williamson et al. 2007). Further, B. cinerea is known to have a large amount of standing genetic variation (Aguileta et al. 2012; Alfonso et al. 2000; Atwell et al. 2015; Baraldi et al. 2002; Fournier and Giraud 2008; Fournier et al. 2002; Giraud et al. 1997, 1999; Ma and Michailides 2005; Munoz et al. 2002; Rowe and Kliebenstein 2007; Staats and van Kan 2012). Several studies have shown that this genetic diversity within $B$. cinerea not only contributes to differential virulence in planta (Calpas et al. 2006; Denby et al. 2004; Rowe and Kliebenstein 2008; Schumacher et al. 2012) but can also contribute to the development of fungicide resistant genotypes (Kretschmer et al. 2009). In addition, recent work has identified natural allelic variants in $B$. cinerea that control the formation

Table 1. Number of traits altered by environmental or genetic variation ${ }^{\mathrm{a}}$

\begin{tabular}{lcccr}
\hline & & \multicolumn{3}{c}{ Fraction of significance } \\
\cline { 3 - 5 } Condition/trait class & Total & $\mathbf{E}$ & Genotype & $\mathbf{G} \times \mathbf{E}$ \\
\hline In vitro & & & & \\
$\quad$ Growth rate & 11 & 6 & 11 & 10 \\
Sporulation density & 10 & 6 & 10 & 6 \\
Sclerotia formation & 10 & 3 & 5 & 3 \\
In planta & 6 & 6 & 6 & 0 \\
Lesion color & 11 & 8 & 6 & 0 \\
Lesion eccentricity & 39 & 39 & 39 & 22 \\
Lesion size & 18 & 18 & 7 & 1 \\
$\quad$ Defense metabolites & & & & \\
\hline
\end{tabular}

${ }^{\text {a }}$ A summary table enumerating the total number of traits measured and the number of traits that showed a significant effect of environment, genotype, genotype $\times$ environment. of sclerotia in response to UV light (Schumacher et al. 2012). However, the role of phenotypic plasticity in $B$. cinerea and how it contributes to overall virulence has largely been unexplored.

To directly determine the relative contribution of plasticity and genetic diversity in $B$. cinerea, we measured multiple traits associated with virulence among 15 genetically distinct isolates of $B$. cinerea under multiple in vitro and in planta conditions. In addition, we also measured the differential accumulation of host-derived defense compounds in response to pathogen attack, to dissect the influence of pathogen plasticity and genetics on host defenses. Our study demonstrates that, while phenotypic plasticity can play a major role for some phenotypes, it often has a smaller effect on the phenotypic diversity of the pathogen than the genetic diversity alone. Further, we found extensive genotype-dependent plasticity for a variety of virulencerelated phenotypes within $B$. cinerea. Using a combination of digital imaging, metabolic profiling, and microscopic surveys, we were able to identify a suite of previously undescribed in planta phenotypes that have yet to be associated with plant-pathogen interactions. Combining all of this data into a correlational network, we were able to uncover novel connections between phenotypes across in vitro phenotyping assays and in planta virulence, showing the potential ability of broad phenotyping surveys to increase our knowledge of plant/pathogen interactions. A combination of hierarchical clustering and principal component analysis (PCA) of phenotypic variation in the traits showed that isolates are largely unique from each other and the differences are driven primarily by in planta lesion traits or responses to individual host defense metabolites in vitro. We believe that this is a unique in-depth description of the virulence-associated phenotypic landscape of a generalist plant pathogen and has implications for future experimental design within plant pathology.

\section{RESULTS}

\section{Effects of environment and genetic variation on $B$. cinerea under in vitro conditions.}

To determine the influence of environment versus genotype within virulence-associated phenotypes across the $B$. cinerea isolates, we first focused on traits measured under in vitro conditions. We chose to challenge a panel of $15 \mathrm{~B}$. cinerea genotypes that have recently been sequenced (Atwell et al. 2015), with a total of 19 different in vitro media that mimic an array of host-based environmental conditions that the fungus is likely to encounter in a variety of potential hosts, including plant-based extracts (e.g., grape and tomato fruit, red and green lettuce leaf, and potato and carrot tuber or root extracts), which vary in their nutrient content and specialized metabolites, and standard potato dextrose agar (PDA) supplemented with either known plant defense compounds (e.g., caffeine, chlorogenic acid, rutin, sinigrin, indol-3-carbinol [ICN], and tannic acid) or plant hormones (e.g., abscisic acid [ABA], gibberellic acid $\left[\mathrm{GA}_{3}\right]$, salicylate $[\mathrm{SA}]$, and jasmonate $\left.[\mathrm{JA}]\right)$. These different in vitro conditions allow us to test the effect of specific aspects of the plant environment on the pathogen, such as resistance to specific plant defense compounds or the ability to utilize defined nutrients, while avoiding the potential of other counteracting effects from other aspects of the host. We have previously used this approach to show that genetic variation in polygalacturonase genes within $B$. cinerea can be linked to the ability to utilize specific plant pectins (Rowe and Kliebenstein 2007). Within every plate across all conditions, we measured hyphal growth, spore formation, sclerotia formation, and other developmental traits on a wide set of media treatments (Table 1; Supplementary Table S2). To first test which traits are effected by environmental or genotypic variance, we applied a linear 
mixed model to each trait and determined the significance and proportion of variance explained $\left(\eta^{2}\right)$ by either the treatment (individual plasticity), the isolate (genetic diversity), and their interaction (Table 1; Fig. 1).

Under in vitro conditions, differences in growth rate across media treatments are largely controlled by the genetic variation among the isolates. For growth on different nutrient media, genetics controlled the largest proportion of variance $\left(\eta^{2}=0.54\right)$, while environment accounted for a smaller but significant proportion of variance $\left(\eta^{2}=0.29\right)$, with a significant interaction between the two $\left(\eta^{2}=0.11\right)$ (Fig. 1). Thus, genetic variation within $B$. cinerea may be maintained by the variation of potential hosts leading to heterogeneous selection pressures on individuals with different abilities to optimize growth in the presence of different nutrient sources. In response to a variety of plant defense compounds and hormones, the variance of growth rate was even more attributable to genetic variation among the isolates $\left(\eta^{2}=0.81 \pm 0.04\right.$ standard error [s.e.]), with relatively little variance due to plasticity (Fig. 1). The exception to this trend was in response to caffeine treatment, in which growth rate appears to be approximately equally controlled by genotype $\left(\eta^{2}=0.43\right)$ and environment (different caffeine concentrations; $\eta^{2}=0.43$ ), which implies that tolerance to different levels of caffeine in $B$. cinerea is driven more by regulated physiological changes than the other host defense compounds tested. The defense compounds tested here are highly lineage-specific within the plant kingdom and, as such, will be heterogeneous among known hosts of $B$. cinerea. The high level of genetic variance altering $B$. cinerea growth in the presence of these compounds shows that $B$. cinerea largely relies upon genetic diversity to cope with these defenses.

In contrast to growth rate, sporulation density across different types of nutrient media was primarily controlled by plasticity $\left(\eta^{2}=0.84\right)$, suggesting that differences in sporulation production are largely due to physiological changes in response to variation in the nutrient sources. Differences for sporulation density in response to individual host defense compounds while still being controlled by genotype also displayed a significant interaction of genotype and environment $\left(\eta^{2}=0.24 \pm 0.03\right.$ s.e. $)$ (Fig. 1). Thus, rather than being purely plastic or controlled by differential genetics, the developmental program for sporulation typically shows an interaction of the two, which allows the isolates to display different sporulation responses to the defense compounds.

In contrast to growth rate and sporulation, the formation of sclerotia across the different nutrient media was most controlled by the interaction between plasticity and genotype $\left(\eta^{2}=0.38\right)$ (Fig. 1), showing that differences among the isolates are dependent on unique physiological responses of each individual to the various nutrient media. The transition to sclerotia in response to plant defense compounds was dichotomous, with the response to caffeine and chlorogenic acid being predominantly influenced

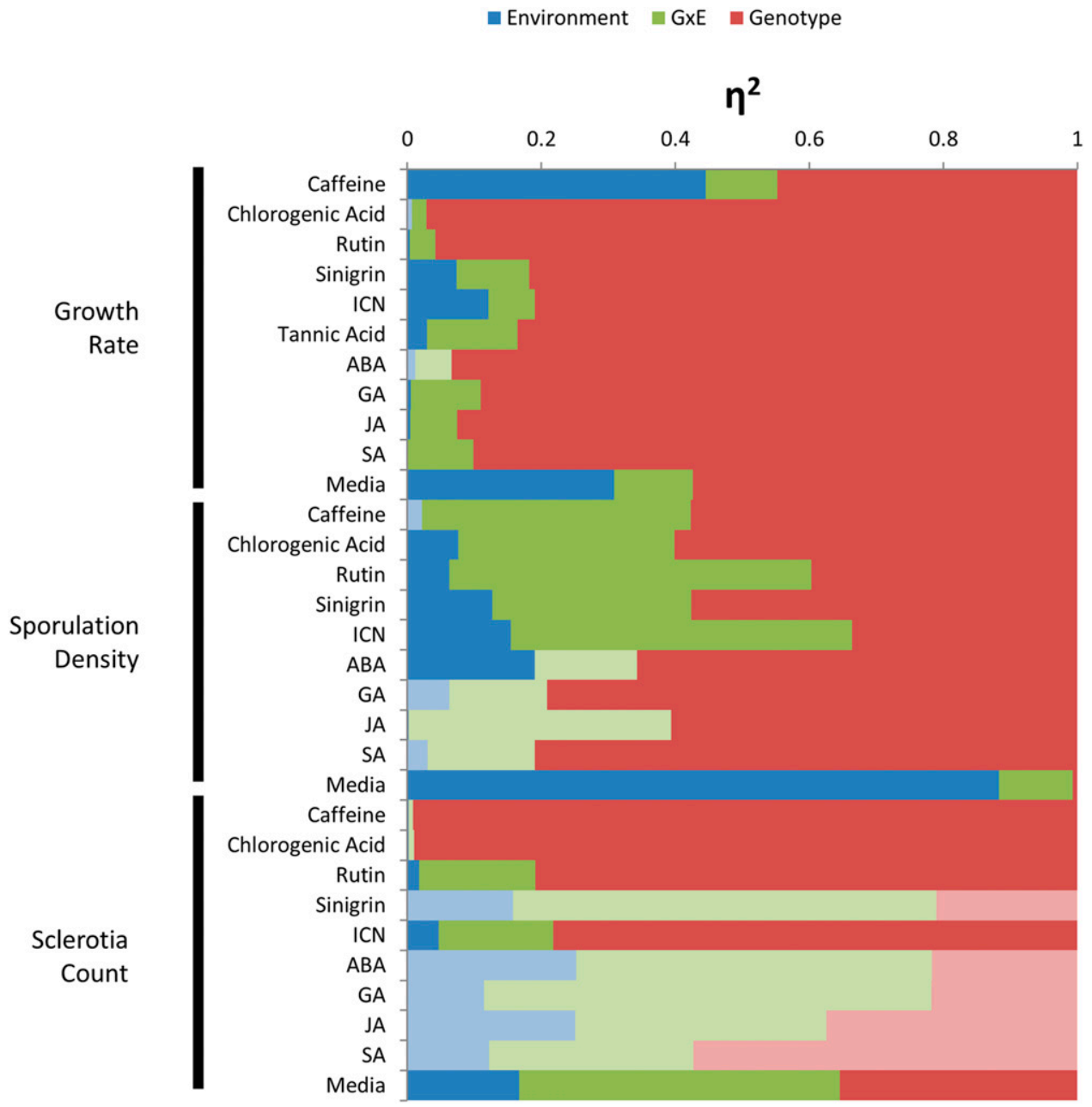

Fig. 1. Proportion of variance $\left(\eta^{2}\right)$ for in vitro traits. Bar graphs depict the relative proportion of phenotypic variance controlled by environment (blue), genetic variation (red), or the the interaction of genotype $\times$ environment (green) for all in vitro phenotypes. Significant variation $(P$ value $<0.05)$ is colored in bold, while nonsignificant variation is at half brightness. Environmental variation within a treatment were estimated by varying either the concentration of the specific compound or the nutrient content of the media. 
by genotype, while sclerotia formation on rutin, sinigrin, and ICN showed significant variation for the genotype $\times$ environment term $\left(\eta^{2}=0.16,0.31\right.$, and 0.16 , respectively). Further, the phenotypic variation attributed to environment or to genotype for media supplemented with known plant hormones was nonsignificant, indicating that $B$. cinerea does not appear to develop sclerotia in direct response to biologically relevant concentrations of plant hormones. Taken together, this argues that the pathogen initiates sclerotia formation in direct response to defense compounds and cannot anticipate host defense responses and physiology by direct detection of plant hormones.

In addition to the $\eta^{2}$ attributed to the genotype and the environment, we also calculated the coefficient of variation $(\mathrm{CV})$ across the model corrected means of each isolate to obtain the genetic $\mathrm{CV}$ and test which phenotypes were displaying more or less population genotypic diversity across individual treatments. CV is a unitless measure of variation calculated by taking the distributions standard deviation divided by its mean and allows for comparisons of distributions across different traits and populations. Growth rate and sporulation density on nutritionally rich media not supplemented with plant defense compounds showed the lowest population genetic CV (Supplementary Table S3), suggesting that, in a nutrient-rich environment, growth and development phenotypes are largely genetically constrained and differences on these traits across the media are largely physiological. In contrast, the developmental transition to sclerotia across these same conditions showed elevated population genetic CV. Since sclerotia are thought to allow the fungus to tolerate a variety of environmental stresses, it is possible that this elevated genetic $\mathrm{CV}$ of sclerotia formation within rich growth media may represent a bet-hedging strategy to anticipate and survive unpredictable changes in this environment.

\section{Effects of environment and genetic variation on $B$. cinerea under in planta conditions.}

The in vitro work allowed us to show that there is extensive genetic diversity and varying levels of plasticity for growth and development of Botrytis cinerea. However, the in planta virulence of the pathogen is driven by an interaction of the genetics between both the pathogen and plant. We used digital imaging to measure the spatial characteristics of active lesions at $72 \mathrm{~h}$ postinoculation (hpi) in the Arabidopsis thaliana ecotype Col-0 and five of its single-gene mutants that have wellcharacterized deficiencies in pathogen defense, including pad3 (camalexin deficient mutant) (Zhou et al. 1999), coil (jasmonate receptor) (Xie et al. 1998), nprl (salicylate receptor) (Cao et al. 1997), tga3, and anac055 (transcription factors involved with response to $B$. cinerea) (Windram et al. 2012). The use of these mutants allows us to survey how genetic shifts in the plant host will influence the pathogen as a model for shifts in host range. By using defined mutants within a single plant genetic background, we have a more defined shift in host genetics than if we were using diverse species or accessions within a species. In addition to spatial characteristics of the lesion, we also measured the concentration of known host defense chemicals in the plants at 72 hpi to provide a measure of host response to the diverse isolates. Since these isolates were grown on the same isogenic plant individuals, we can largely ignore differences in the genetics of the host and treat them as different, albeit dynamic, environments for the fungal pathogen. Hence, plant treatment represents the individual plasticity of the fungus across these in planta growing conditions as created by the different plant genotypes.

In addition to controlling potential genetic variation from the host through experimental design, we used a linear mixed model to control for random variation due to the temporal experimental replicates and growing blocks. Applying this method to the spatial characteristics of lesion, a significant proportion of the variance within the linear models was explained by the host background $\left(\eta^{2}=0.33 \pm 0.02\right.$ s.e.) (Fig. 2 ). This implies that plasticity of the pathogen to genetic variation in the host is an important characteristic during in planta interactions, since the host background is the environment from a pathogens perspective. Genetic variation within $B$. cinerea also played a significant role in the interaction, generally explaining a significant and approximately equal amount of variation $\left(\eta^{2}=0.40 \pm 0.02\right.$ s.e. $)$ among the spatial characteristics, including lesion area, maximum radius, and perimeter (Fig. 2). Genotype and environment also significantly controlled lesion color traits, as measured by the proportion of yellow and green pixels in the residual leaf, and lesion eccentricity (deviation from circularity) (Fig. 2). Neither of these traits has been previously characterized as a component of the plant-pathogen interaction. While the eccentricity of the lesion appears to differ drastically from the other spatial characteristics in that a larger proportion of the variance is explained by the interaction between individual plasticity and genetic diversity, this variation was not significant (Fig. 2). The equal contributions of plasticity versus genotype under in planta conditions imply that each contributes equally to the trait, which is in contrast to the in vitro settings that were more dominated by genetic diversity among the isolates (Figs. 1 and 2).

The contributions of plasticity and genetic variation among the $B$. cinerea isolates' ability to induce host defense compounds in planta were far more varied than lesion spatial characteristics across the population. Differences in aliphatic glucosinolate concentrations were primarily dominated by plasticity $\left(\eta^{2}=0.42 \pm 0.07\right.$ s.e. $)$, while differences due to genotype of the pathogen were often nonsignificant. The remaining defense compounds showed a similar pattern, with the exception of the proportion of $4 \mathrm{MOI} 3 \mathrm{M}$ to the total indole glucosinolates and camalexin, which both showed a large and significant proportion of variance explained by the $B$. cinerea genotype $\left(\eta^{2}=0.45\right.$ and 0.37 , respectively). Intriguingly, $4 \mathrm{MOI} 3 \mathrm{M}$ and camalexin are the host defense compounds most frequently associated with pathogen defense in Arabidopsis (Ferrari et al. 2003, 2007; Kliebenstein et al. 2005). Thus, identifying the loci in the $B$. cinerea genome that differentially stimulate these defense compounds could provide information about the interaction of host and pathogen.

The CV of in planta phenotypes also showed that population phenotypic diversity was extremely variable for in planta phenotypes. On average, individual host defense metabolites showed statistically significant higher $\mathrm{CV}$ for in planta phenotypes $(\mathrm{CV}=0.43 \pm 0.04$ s.e. $)$ than spatial descriptions of the lesion ( $\mathrm{CV}=0.23 \pm 0.01$ s.e.) ( $t$ test $P<0.0001, n=437)$. This higher genetic variation in $B$. cinerea for the ability to alter plant metabolic phenotypes in comparison with lesion development could suggest that there are fewer biological inputs for a single plant response (i.e., metabolite) in comparison with the development of the entire lesion.

\section{Evidence of genetic variation altering qualitative traits.}

The in vitro growth data showed that there is significant influence from plasticity and genotype in the growth habits of the $B$. cinerea isolates for a variety of traits. Further, the above data showed that macroscopic measures of $B$. cinerea-virulence in planta were also dependent upon the genetic variation present within the pathogens population. To test if there is genetic variation at the microscopic level of the pathogens development in planta, we measured the growth of the different isolates using trypan blue-stained A. thaliana leaves at $72 \mathrm{hpi}$ for the Col-0 accession and its pad 3 mutant, using three independent replicates of each isolate. This showed a wide range 
of genetically dependent microscopic growth traits across the isolates that had not previously been noted, the most striking of which is the density of hyphae within the mycelial mat in planta (Fig. 3), where isolates range from having a diffuse hyphal arrangement (e.g., B05.10 [Fig. 3C]) to a densely packed hyphal arrangement (e.g., Rose [Fig. 3M]). Additionally, five of the isolates with diffuse hyphal patterns send out infrequent exploratory hyphae that stretch beyond the primary mycelial mat

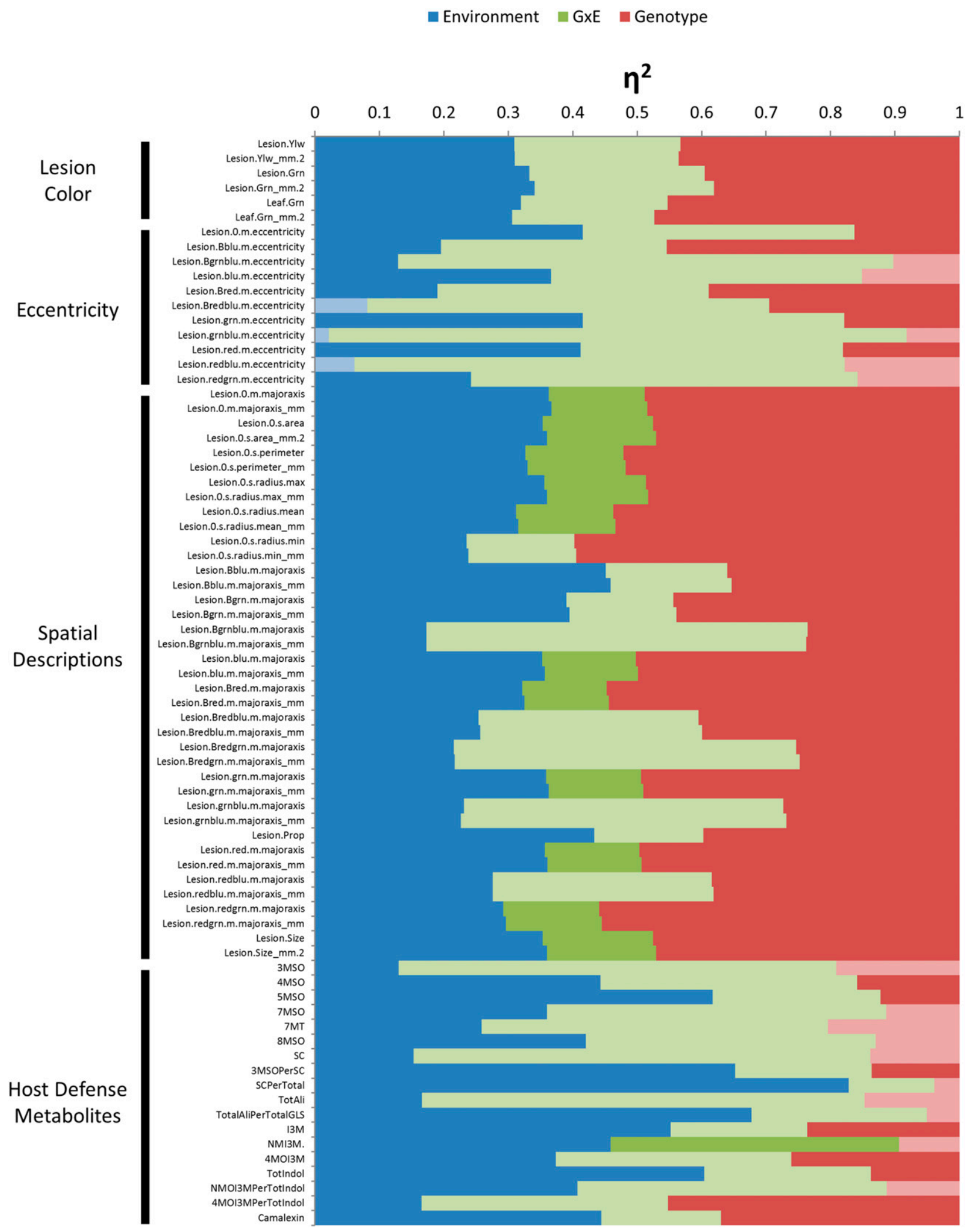

Fig. 2. Proportion of variance $\left(\eta^{2}\right)$ for in planta traits. Bar graphs depict the relative proportion of phenotypic variance dedicated to environment (blue), genetic variation (red), or genotype $\times$ environment interactions (green) for all in planta phenotypes. Significant variation $(P$ value $<0.05)$ is colored in bold, while nonsignificant variation is at half brightness. Environmental variation within a treatment were estimated by infecting several single-gene mutants known to affect the plant innate immune response. 
(Fig. 3), as best represented by the PepperSub isolate (Fig. 3L). Eleven of the isolates also have a tendency to form dense vegetative bodies (DVB) in planta that appear to be developing microsclerotia (Supplementary Fig. S1). Leaves were incubated for $4 \mathrm{~h}$ in $10 \mathrm{~N} \mathrm{NaOH}$, with agitation, to test if these DVB were crystalized oxalate deposited by the fungus. These DVB did not dissolve or shrink in size in response to the $\mathrm{NaOH}$ treatment, suggesting that they are not deposits of oxalate. Another genetically dependent microscopic phenotype observed was a demonstrated and a reproducible preference for hyphal growth along the vasculature of the host for five isolates, as can be seen in Supersteak (Fig. 3B). In contrast, other isolates showed no such bias in growth pattern and were predominantly radial in pattern. This bias toward vascular growth could indicate that these isolates preferentially infect specific host cell types or follow metabolic gradients. All of these phenotypes were identical on the
Col-0 and pad3 Arabidopsis genotypes suggesting that they are largely controlled by the pathogen genetics and are largely not plastic in nature. Thus, there appears to be genetic variation within Botrytis controlling microscopic growth traits in planta. As these phenotypes were largely qualitative and difficult to measure, they were not included in the below analysis of quantitative variation.

\section{Links between in vitro and in planta virulence-related traits among $B$. cinerea isolates.}

The combination of virulence-related traits measured in vitro and in planta across a diverse set of isolates allows us to test if there is any detectable relationship between these phenotypes. Evidence of associations will help to understand how to interpret the in vitro work with regards to how this information pertains to in planta infections. To look for associations

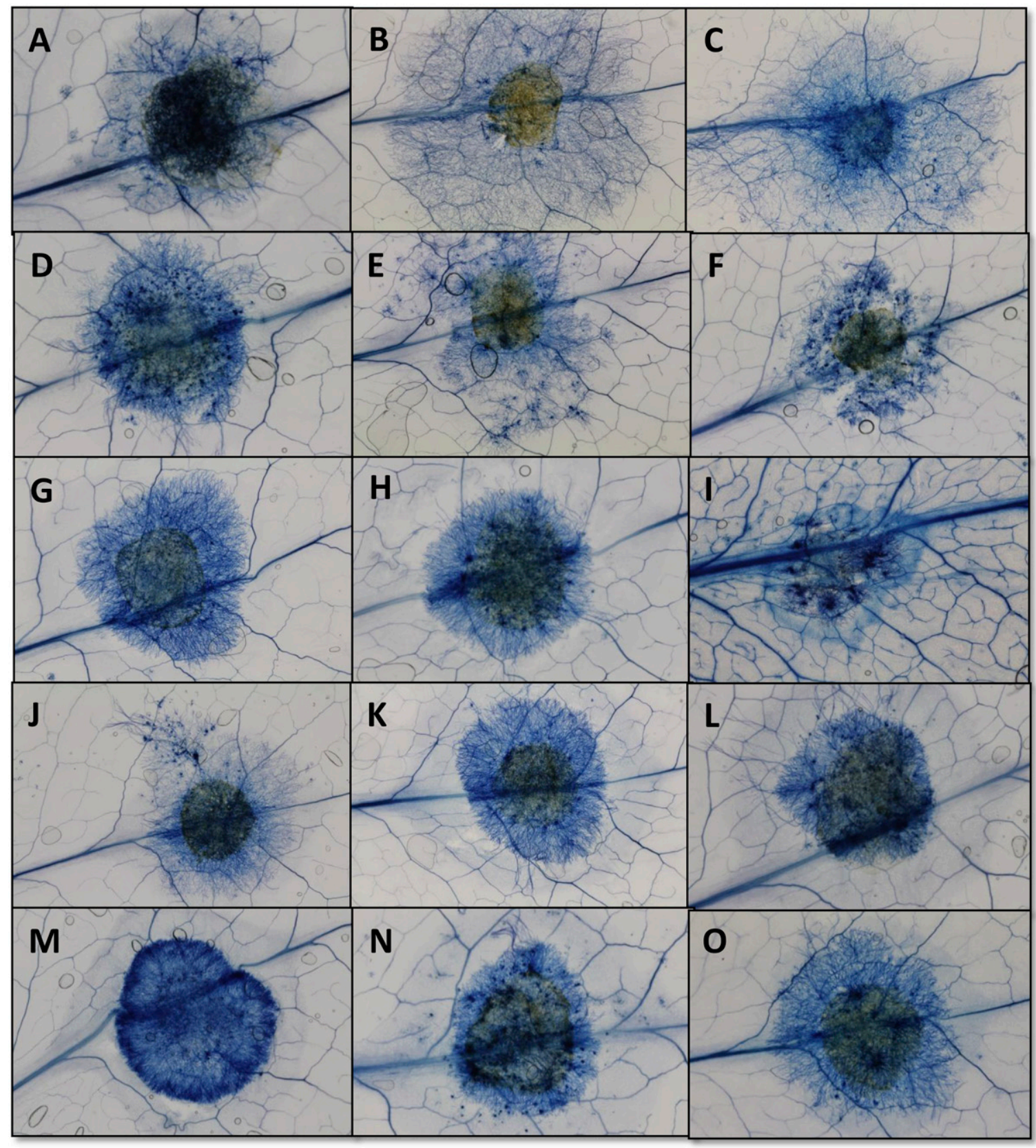

Fig. 3. Qualitative traits in trypan blue-stained infected leaves of Arabidopsis thaliana. Representative examples of infected Col-0 leaves at $72 \mathrm{~h}$ postinoculation and stained with trypan blue. The samples illustrate the phenotypic diversity observed in A, acacia, B, Apple517, C, B05.10, D, Davis navel, E, Fresa S.D., F, Gallo1, G, geranium, H, grape, I, Katie tomato, J, Noble rot, K, pepper, L, PepperSub, M, Rose, N, Supersteak, and O, UK Razz. 
between these phenotypes, we constructed a phenotypic correlation network matrix to link all quantitative in vitro and in planta traits (Fig. 4; Supplementary Table S4). The network revealed several interesting relationships among the in vitro and in planta traits across the $B$. cinerea isolates. The most consistent set of correlations among the traits is the positive correlations among many of the different spatial aspects of lesion size, such as lesion area, perimeter, major axis, and radius. Interestingly, many of these in planta spatial lesion descriptors were positively correlated with in vitro growth rate on media supplemented with plant hormones. In contrast to these positive correlations, there were a number of negative correlations between these in planta spatial descriptors and in vitro sclerotia formation on media with plant hormones. This suggests that there may be a potential trade-off between in planta virulence and sclerotia formation that could protect the pathogen from harsh environmental conditions. Interestingly, in vitro traits on media supplemented with disease-resistance compounds showed little to no correlation with in planta lesion development. This is likely because the commercially available defense compounds we were able to obtain are not present within Arabidopsis. However, these results show that it is possible to use genetic variation within the pathogen to link in vitro and in planta phenotypes potentially providing the ability to dissect the pathogens contribution to the developing lesion.

Two aspects of the in planta lesion description were largely independent of the spatial descriptions of the lesion. These were the eccentricity, or deviation of the lesion from circularity, and the color of the lesion (Fig. 4). Both of these traits displayed genotypic and environmental impacts but showed no correlation to the spatial descriptors of the lesion, indicating that the genetic variation in the pathogen affecting these traits is largely separate from the genetics controlling general lesion size. While these phenotypes were not correlated with the other spatial descriptions of the lesion, both eccentricity and lesion color were largely negatively correlated with in vitro growth and sclerotia development on media with plant hormones. This analysis shows that it is possible to use digital imaging of in planta lesion development to obtain new measurements of lesion development that have been previously uncharacterized and are genetically independent, previously described, virulence-related phenotypes.

Similar to lesion color and eccentricity, the plants' response to infection as measured by the accumulation of various in planta defense compounds (glucosinolates and camalexin) was uncorrelated within any of the visible lesion measurements (Fig. 4). Thus, the plants' measured response to genetic diversity in the pathogen is not driving the variation in lesion formation in this population. The strongest link between accumulation of in planta host defense compounds and in vitro phenotypes was the correlation between in planta aliphatic glucosinolates and in vitro sporulation density on media supplemented with plant hormones (Fig. 4). In planta indolic glucosinolate accumulation was most tightly linked with in vitro sclerotia count on media supplemented with plant hormones (Fig. 4). This suggests that there may be a link between the genetic architecture within $B$. cinerea that controls

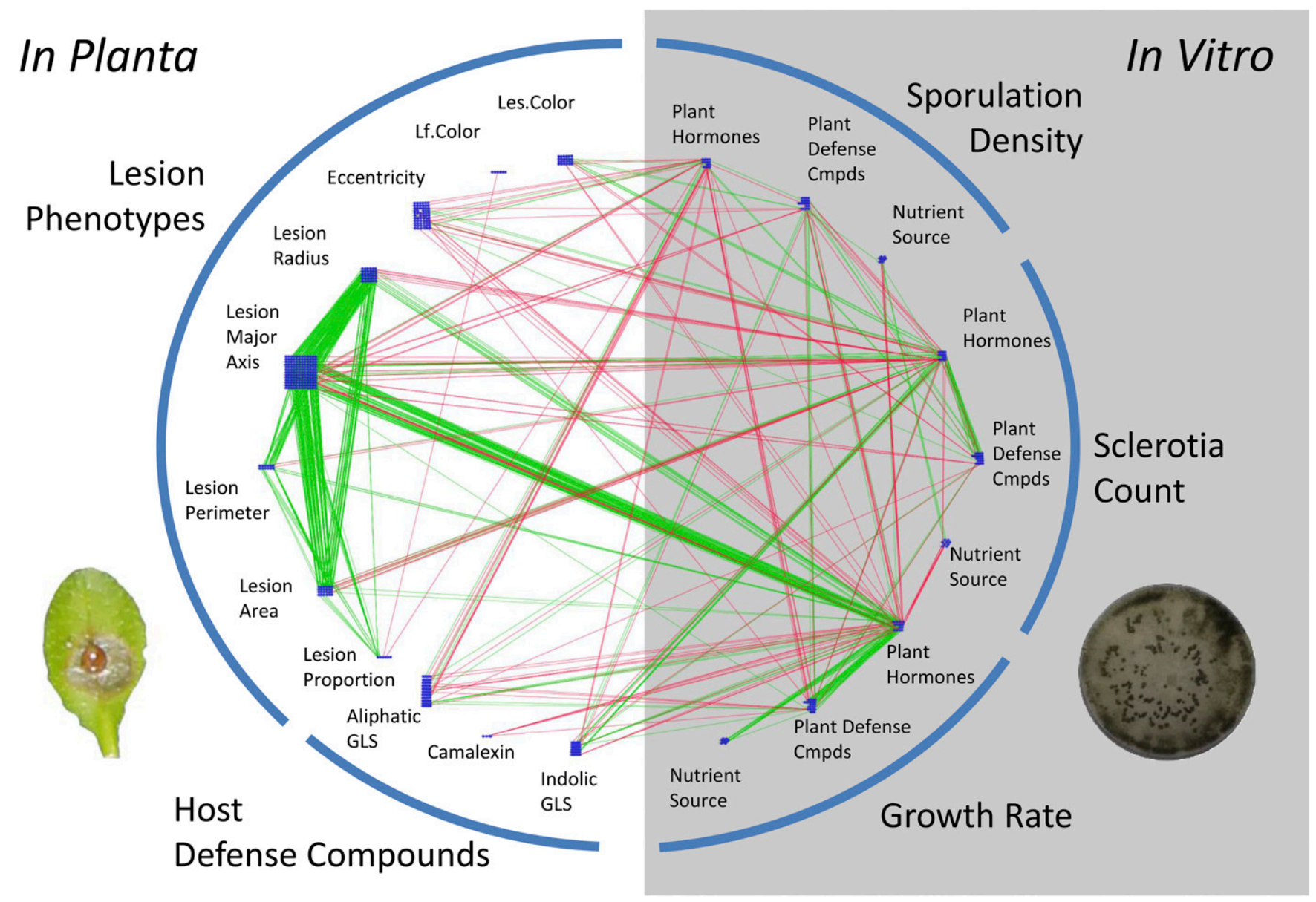

Fig. 4. Correlation network for in vitro and in planta traits. A Pearson's correlation network of model corrected phenotypic means for all traits measured in this study. In planta traits are listed on the left while in vitro traits are in the shaded background on the right. All edges represent a Pearson's correlation $>0.95$, where green edges represent positive correlations and red edges represent negative phenotypes. 
developmental responses to individual plant hormones and how the A. thaliana host responds to these distinct isolates. Thus, genetic variation in $B$. cinerea may affect the interplay between plant and pathogen at the signaling level. Further work is needed to elucidate the biological processes that underlie this phenomenon.

\section{Each isolate is a unique combination of traits.}

While correlational networks illustrate the association among traits and imply an interrelated underlying genetic architecture across a wide range of in vitro and in planta characteristics, it does not provide information on how phenotypically distinct the isolates are from each other and which traits may drive most of these differences among the isolates. To investigate the distinctiveness of the isolates, we conducted a hierarchical clustering analysis using all quantitative traits. Clustering analysis suggested the presence of at least two separate clades of the
B. cinerea isolates, with Apple517 presenting as a major outlier (Fig. 5A). However, a bootstrap analysis showed that the majority of branches within the tree had no statistical support, which indicates that these isolates are not showing evidence of trait covariation, as this would have been evidenced by a more structured dendrogram. This demonstrates that the isolates are phenotypically equidistant from each other and do not form distinct phenotypic clades, suggesting that each individual isolate represents a unique sample of the potential traits that we measured. Thus, each isolate is a unique individual and there is little evidence for clonal selection in terms of traits diversity.

As an alternate description of traits relatedness, we conducted a PCA of all the traits across the isolates (Fig. 5B). This analysis showed that the first two components only accounted for 32 and $10 \%$ of the variance among the isolates, which agrees with the bootstrap analysis in the hierarchical clustering results, suggesting a high level of traits admixture among the

\section{A Cluster dendrogram with BP values (\%)}

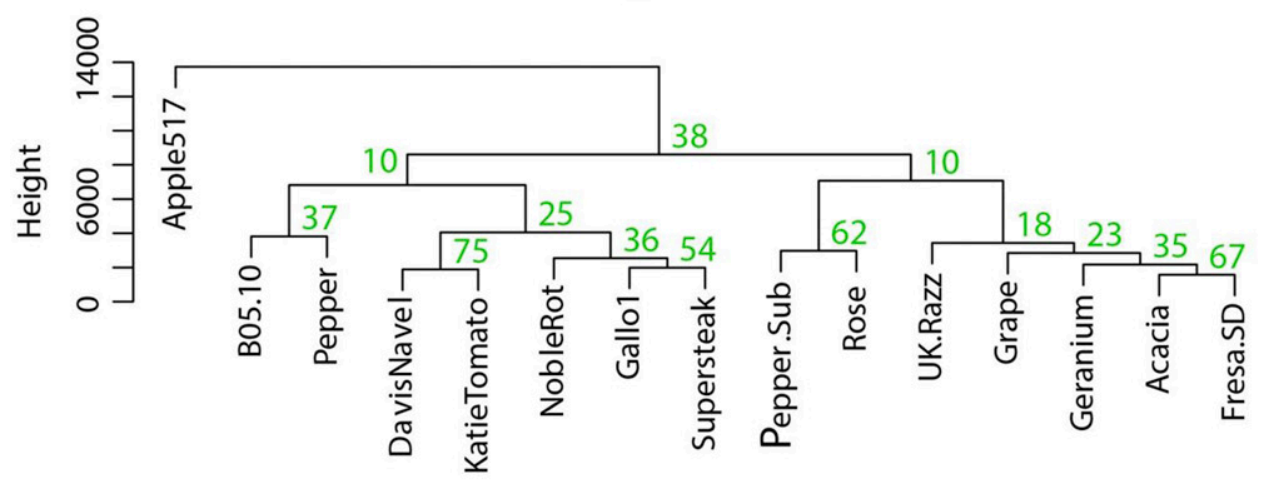

Distance: euclidean

Cluster method: ward

B

Individuals factor map (PCA)

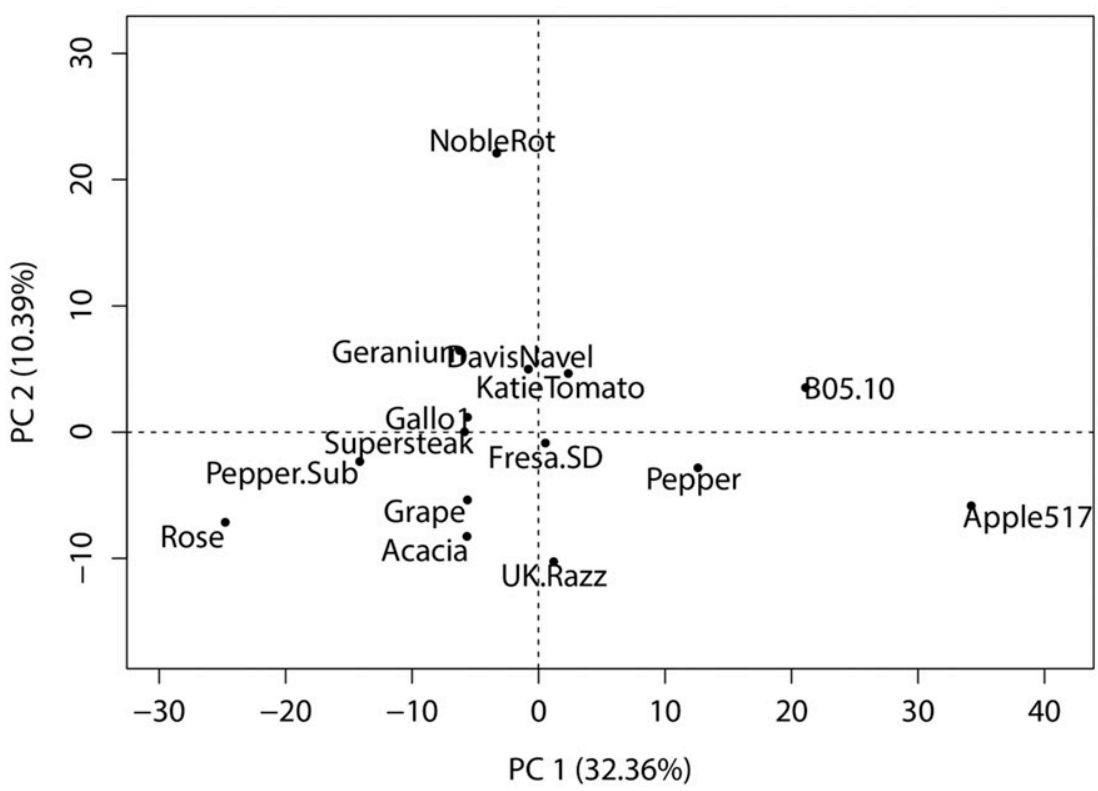

Fig. 5. Hierarchical clustering and principal component analysis (PCA) of traits. Plots of how phenotypic variation for the measured traits illustrates the distinctness among the isolates. A, Dendrogram illustrating the hierarchical clustering of the isolate Euclidean distance matrix for all traits. Green numbers indicate the bootstrap support for each node. B, PCA showing the separation of isolates across the first two principal components for all quantitative traits. 
isolates (Fig. 5). The isolates were broadly distributed using these two components without obvious clustering or trends among the isolates. The traits driving the first principal component vector were those associated with how the isolates differ in their in planta spatial descriptions of lesion size (Supplementary Table S5). The second principal component was driven primarily by the isolates' ability to alter in planta glucosinolate concentrations in the A. thaliana host as well as their growth rate on media supplemented with chlorogenic acid. The traits dominating these components showed the highest environmental and genotypic effects under in planta conditions. Thus, it appears that both plasticity and genotype in $B$. cinerea is driving the trait differences among the isolates. These are the key traits that would be required to show genetic differentiation in a pathogen whose wide host range is nearly the entire Viridiplantae and that also has the capacity to exist as a saprophyte.

\section{DISCUSSION}

\section{Both plasticity and genotype contribute to virulence-related traits in $B$. cinerea.}

As a generalist fungal pathogen, $B$. cinerea should be a good model for exploring phenotypic plasticity and trait diversity in a plant pathogen. Our analysis of in planta and in vitro traits in a population of $B$. cinerea showed that the majority of traits were controlled by a mixture of environment, genotype, and their interaction (Figs. 1 and 2). Interestingly, the observed traits showed a differential contribution of the relative fractions across these three factors that may be linked to biology and the underlying genetic architecture. For example, relative sporulation density on different in vitro nutrient media is largely plastic (i.e., environmentally responsive), while the relative sporulation to individual plant defense compounds is predominantly genotype-dependent (Fig. 1). This may indicate that there is more heterogeneous variation in plant defense compounds across the potential plant hosts in comparison with the relative nutrient content of these hosts. In addition, several traits showed a large proportion of phenotypic variance explained by the interaction of genotype $\times$ environment, such as lesion eccentricity (Fig. 2). In this case, the isolates show differentially plastic responses when confronted by a range of plant genotypes. From a systems perspective, this suggests that these complex traits are controlled by complicated molecular mechanisms that sense, process, and respond to environmental inputs in a manner that is largely specific to each pathogen genotype. Further, this genetics-dependent plasticity from the pathogen is also important for understanding host resistance, as pathogen genetic diversity and plasticity also affected plant host metabolic defenses (Fig. 2). Thus, any full molecular assessment of pathogenicity in the $B$. cinerea-plant pathosystem must include an array of diverse isolates (Denby et al. 2004; Iacomi-Vasilescu et al. 2008; Kliebenstein et al. 2005; Rowe and Kliebenstein 2007; 2008; Schumacher et al. 2012; Staats et al. 2007; Zhang et al. 2003).

\section{Isolates are phenotypically unique members of a single diverse population.}

Both the boot-strap analysis within the hierarchical clustering dendrogram and trait weights from the PCA illustrated that each isolate is distinct across the differing environmental conditions (Fig. 5). Further, the linear mixed models from the in vitro and in planta experiments showed that these trait differences are largely due to genetic variation among the 15 isolates (Figs. 1 and 2). In combination with previous genetic studies, this suggests that $B$. cinerea is not reproducing in a clonal manner and, instead, is allowing for extensive recombination from either meiotic or mitotic processes to generate a large pool of diversity (Amselem et al. 2011; Fournier and Giraud 2008;
Fournier et al. 2002; Giraud et al. 1997, 1999; Ma and Michailides 2005; Munoz et al. 2002; Rowe and Kliebenstein 2007; Staats and van Kan 2012). This genetic diversity and its phenotypic consequences among the isolates could be what allows $B$. cinerea to infect such a large number of potential plant hosts. This agrees with previous reviews that have argued that pathogens with a large amount of standing genetic diversity and a large effective population size, like $B$. cinerea, have the potential to be among the most difficult to control agricultural pests (McDonald and Linde 2002). Further, generalist pathogens with this high level diversity may contain a reservoir of genetic variation allowing the pathogen to quickly overcome horticultural techniques designed to control outbreaks, such fungicides. This argues that $B$. cinerea is both an excellent model for studying how a pathogen's genetics affect virulence as well as having new potential practical applications.

\section{Identifying new traits and links between traits.}

By analyzing many different genetic isolates of $B$. cinerea, we were able to identify several novel traits within the species under in planta conditions. For instance, qualitative traits identified in trypan blue-stained leaves, including differences in hyphal density, exploratory hyphae, DVB, and growing preferences for host vascular tissues, have not been previously described to our knowledge (Fig. 3). In addition to the microscopic investigation, our digital analysis approach also identified new traits not previously linked to plant-pathogen interactions. For example, lesion eccentricity was not correlated to spatial descriptions of the lesion (Fig. 4). This tells us that the shape of the lesion is not necessarily related to its size (area, perimeter, major axis, and radius) and could provide new information about isolate virulence, while the other spatial descriptions of the lesion are related and provide similar information. The fact that these traits are genetically variable suggests that the underlying molecular mechanisms may be accessible using genome-wide association mapping or other natural variation approaches. Once the underlying molecular mechanisms are identified, it will be possible to test how these novel traits influence the pathogenhost interactions.

\section{Future work.}

With the advent of high-throughput genomic sequencing and rapid techniques for genome-wide genotyping, there is a dire need to develop better methods for high-throughput phenotypic screening and new theoretical framework to explain trait relationships, to better understand both the genotype-phenotype and phenotype-phenotype connections. In this study, we attempted to survey several high-throughput traits to better understand what traits may contribute to virulence in $B$. cinerea and what traits may be of interest for genetic mapping. As such, we have already begun measuring multiple in planta traits in a population of Arabidopsis thaliana host infected with four of the phenotypically diverse isolates studied here, as well as a collection of 96 isolates of $B$. cinerea on several $A$. thaliana mutants in known host defense pathways. This study has been informative to help us understand what phenotypes are measurable and worth measuring for genetic mapping in both the host and pathogen in the Arabidopsis-Botrytis pathosystem.

\section{MATERIALS AND METHODS}

Germplasm maintenance.

A collection of 15 Botrytis cinerea isolates was selected based on the geographic location in which they were collected and the plant material from which they were collected (Table 1). Several of these isolates have been previously characterized for pectin degradation and camalexin tolerance 
(Kliebenstein et al. 2005; Rowe and Kliebenstein 2007). Isolates were maintained on $1 \times$ PDA and were incubated at room temperature (approximately $22^{\circ} \mathrm{C}$ ) for up to 10 days. New plates were inoculated with an agar plug containing the leading edge of active mycelia or, alternatively, from $-80^{\circ} \mathrm{C}$ freezer stocks of spores.

\section{Monitoring pathogen characteristics under in vitro conditions.}

To test if $B$. cinerea traits are altered by plasticity or genetic variation in response to components of host defense, all 15 $B$. cinerea isolates were monitored for growth rate, sporulation density, and sclerotia count in response to a variety of in vitro media treatments. All plates were inoculated using a $1 \times 1 \times$ $1 \mathrm{~cm}$ block of agar cut from the leading edge of actively growing mycelia on PDA and were incubated on the bench top at room temperature (approximately $22^{\circ} \mathrm{C}$ ) with light cycles (fluorescent and daylight) of approximately $16 \mathrm{~h}$. All cultures were grown with three biological replicates and were monitored for mycelial growth (measured every $12 \mathrm{~h}$ ) over the initial $72 \mathrm{hpi}$, and sporulation density and sclerotia count were measured at 7 dpi.

Media treatments included a several-defined and undefined agar media as well as standard media supplemented with different compounds associated with plant defense and biological control. Defined media included the BSMmin media $(118 \mu \mathrm{M}$ $\mathrm{NaNO}_{3}, 63 \mu \mathrm{M} \mathrm{K}_{2} \mathrm{HPO}_{3}, 81 \mu \mathrm{M} \mathrm{MgSO}+7 \mathrm{H}_{2} \mathrm{O}, 134 \mu \mathrm{M} \mathrm{KCl}$, $11.1 \mathrm{mM}$ glucose, 2\% phytagar) and Gamborg's B5 Salts supplemented with $2.0 \%$ glucose and $2 \%$ phytagar. Undefined nutrient media included a variety of plant juice extracts including Lactuca sativa 'Green Leaf' $(0.5 \times$ fresh leaf juice), Lactuca sativa 'Red Leaf' (0.5× fresh leaf juice), Daucus carota $(0.25 \times$ root juice [Odwalla Inc., Half Moon Bay, CA, U.S.A.]), Solanum tuberosum (PDA [BD Difco, Franklin Lakes, NJ, U.S.A.]), Malus domestica $(0.25 \times$ fruit juice [Santa Cruz Natural, Inc., Chico, CA, U.S.A.]), Citrus spp. (0.25× fruit juice [Langer's Juice Company, City of Industry, CA, U.S.A.]), Solanum lycopersicum $(0.15 \times$ 'low sodium' fruit juice concentrate [Campbell's Soup Company, Camden, NJ, U.S.A.]), Vitis vinerfera Concord $(0.25 \times$ fruit juice concentrate [Santa Cruz Natural, Inc.]). Diluted plant extracts were combined with $1.5 \%$ phytagar, were autoclaved for $20 \mathrm{~min}$ at $121^{\circ} \mathrm{C}$, and were poured into petri plates.

The effect of individual plant defense-related compounds was tested using petri plates containing PDA, supplemented with biologically relevant concentrations of purified known plant disease-resistance compounds, plant hormones, and a synthetic fungicide used to control Botrytis infection. Plant disease-resistance compounds used in this study included ICN $(0,0.1,0.2,0.5 \mathrm{mM})$, sinigrin $(0,0.1,1,5 \mathrm{nM})$, caffeine $(0$, $0.05,0.1,0.5 \%)$, rutin $(0,0.125,0.25 \mathrm{mM})$, chlorogenic acid $(0$, $0.25,1.0,2.0 \mathrm{mM})$, tannic acid $(0,50,100 \mathrm{mg} / \mathrm{ml})$. Plant hormones used in this study included $\mathrm{ABA}(0,0.5,1,10 \mathrm{nM}), \mathrm{JA}$ $(0,50 \mathrm{ng} / \mu \mathrm{l}), \mathrm{SA}(0,0.7,3.5,10.8 \mathrm{nM})$, and $\mathrm{GA}_{3}(0,0.1,0.5$, $10 \mathrm{nM})$. The synthetic fungicide used in the study was fenhexamide $(0,3.125,6.25,12.5,25 \mu \mathrm{g} / \mathrm{ml})$. Inoculated cultures were incubated on the bench top at room temperature (approximately $22^{\circ} \mathrm{C}$ ) with light (fluorescent and day) cycles of approximately $16 \mathrm{~h}$. All cultures were grown with three biological replicates and were monitored for mycelial growth (measured every $12 \mathrm{~h}$ over $72 \mathrm{~h}$ ) as well as sporulation density and sclerotia size and number at $7 \mathrm{dpi}$.

\section{Monitoring pathogen characteristics under in planta conditions.}

To investigate in planta traits, a variety of virulenceassociated traits were monitored in infected leaves of
Arabidopsis thaliana accession Col-0 and several single-gene mutants with deficiencies in known defense response pathways or compounds (pad3, coil, nprl, anac055, tga3). Infections were completed as previously described (Denby et al. 2004). Briefly, plants were grown in controlled environments with 10-h day lengths in a complete block design, with two experimental replicates separated by two weeks and four biological replicates in each. Detached leaves of five-week-old plants were collected and inoculated with $4 \mu \mathrm{l}$ of $B$. cinerea spores suspended in $0.5 \times$ grape juice (fruit juice concentrate from Santa Cruz Natural, Inc.) at a concentration of 10 spores per microliter for each of the isolates. Leaves were incubated on $1.0 \%$ phytagar for $48 \mathrm{~h}$ at room temperature under constant light. Pictures of infected leaves were taken at 72 hpi and individual interactions were measured using an automated image analysis pipeline, using the CRImage package in the R statistical environment to measure lesion spatial characteristics (area, perimeter, eccentricity, major axis, the maximum, minimum, and average radius, and the proportion of the leaf), color of the leaf and lesion, and a variety of Harlick pixel transformations of the spatial descriptions. Leaf color was measured by transforming pixels from red-green-blue to hue-saturationvalue color space and counting the area of yellow- and greenhued pixels as a proportion of total leaf area of the residual leaf outside the lesion. Leaves were also collected at this time for high-performance liquid chromatography analysis of the known Arabidopsis defense compounds camalexin and glucosinolates. Camalexin was normalized to the lesion perimeter, as it is induced the pathogen front during infection, while glucosinolates were normalized to the remaining leaf area, since they are produced prior to pathogen attack. Extraction and quantification of these defense compounds were conducted as previously described (Kliebenstein et al. 2001a and b, 2005).

In addition, several qualitative phenotypes were studied in trypan blue-stained leaves of the Arabidopsis ecotype Col-0 at 72 hpi for all 15 isolates. Leaves were infected as described above and were stained as previously described (Rate et al. 1999; van Wees 2008). Isolate-specific differences were observed in the relative hyphal density, DVB, and the tendency to develop exploratory hyphae and to leaf vasculature.

\section{Statistical methods.}

All statistical methods were implemented in the $\mathrm{R}$ statistical software package and were conducted separately for each trait $(\mathrm{R}$ Development Core Team 2015). Significance of the genomic and treatment effect, the proportion of variance explained $\left(\eta^{2}\right)$, and the estimated LSMeans for each isolate within a treatment was determined using one of the following linear models for each trait.

Image and inplant chemical defense-related traits: $Y_{i j}=E+B+P_{i}+F_{j}+P_{i}: F_{j}$

Media supplemented with plant hormones or plant defense chemicals: $Y_{i j}=C_{i}+F_{j}+C_{i}: F_{j}$

Defined and undefined media (all treatments run in a single model): $Y_{i j}=M_{i}+F_{j}+M_{i}: F_{j}$,

where $\mathrm{E}$ and $\mathrm{B}$ represent the random effects of the experiment and block, respectively, and the fixed effects are represented by the plant genotype $(\mathrm{P})$, the fungal isolate genotype $(\mathrm{F})$, the concentration of the compound in the media $(\mathrm{C})$, and the type of nutrient media $(\mathrm{M})$ the isolates were grown on. The LSMean of each trait within a treatment and associated for each fixed and random effect was calculated using the 'doBy' package in R, which calls upon the 'nlme' package allowing for the use of adaptive Gaussian quadrature to minimize the effects of deviations from normality and the calculation of variances using REML (Pinheiro and Chao 2006). The LSMean of each trait was used for further downstream statistical analyses. 
Exploring the relationships between traits and isolates was accomplished using several methods. A PCA was conducted using the $\mathrm{R}$ package 'FactoMineR' to determine which isolates are the most similar to one another (Le et al. 2008). The vector loadings of the traits were used to determine what phenotypes vary the most among the selected 15 isolates. In addition, a hierarchical clustering of the scale model-corrected traits was conducted among the isolates and scaled phenotypes, using the default settings of the 'pvclust' package in R (Suzuki and Shimodaira 2006). Co-occurrence among the traits using only pair-wise complete observations with Pearson's productmoment correlations and the trait network was determined using correlations $>0.95$ in Cytoscape (Smoot et al. 2011).

\section{ACKNOWLEDGMENTS}

We thank the laboratories of K. Denby (University of Warwick), J. Van Kan (Wageningen University), H. Rowe and M. Vivier (Institute of Wine Biotechnology), E. Mitcham (University of California -Davis), and G Loake (University of Edinburgh) for generously sharing the isolates used in this study. Funding for this work was provided by the National Science Foundation award IOS 1339125, the United States Department of Agriculture National Institute of Food and Agriculture, Hatch project number CA-D-PLS-7033-H, and by a Danish National Research Foundation (DNRF99) grant to D. J. Kliebenstein, and by the Jastro Shields and Graduate Student Researcher Awards from the University of CaliforniaDavis, Plant Science Department to J. A. Corwin.

\section{LITERATURE CITED}

Agrawal, A. A. 2001. Phenotypic plasticity in the interactions and evolution of species. Science 294:321-326.

Aguileta, G., Lengelle, J., Chiapello, H., Giraud, T., Viaud, M., Fournier, E., Rodolphe, F., Marthey, S., Ducasse, A., Gendrault, A., Poulain, J., Wincker, P., and Gout, L. 2012. Genes under positive selection in a model plant pathogenic fungus, Botrytis. Infect. Genet. Evol. 12:987-996.

Alfonso, C., Raposo, R., and Melgarejo, P. 2000. Genetic diversity in Botrytis cinerea populations on vegetable crops in greenhouses in south-eastern Spain. Plant Pathol. 49:243-251.

Amselem, J., Cuomo, C. A., van Kan, J. A. L., Viaud, M., Benito, E. P., Couloux, A., Coutinho, P. M., de Vries, R. P., Dyer, P. S., Fillinger, S., Fournier, E., Gout, L., Hahn, M., Kohn, L., Lapalu, N., Plummer, K. M., Pradier, J. M. Quévillon, E., Sharon, A., Simon, A., ten Have, A., Tudzynski, B., Tudzynski, P., Wincker, P., Andrew, M., Anthouard, V., Beever, R. E., Beffa, R., Benoit, I., Bouzid, O., Brault, B., Chen, Z., Choquer, M., Collémare, J., Cotton, P., Danchin, E. G., Da Silva, C., Gautier, A., Giraud, C., Giraud, T., Gonzalez, C., Grossetete, S., Güldener, U., Henrissat, B., Howlett, B. J., Kodira, C., Kretschmer, M., Lappartient, A., Leroch, M., Levis, C., Mauceli, E., Neuvéglise, C., Oeser, B., Pearson, M., Poulain, J., Poussereau, N. Quesneville, H., Rascle, C., Schumacher, J., Ségurens, B., Sexton, A., Silva, E., Sirven, C., Soanes, D. M., Talbot, N. J., Templeton, M., Yandava, C., Yarden, O., Zeng, Q., Rollins, J. A., Lebrun, M. H., and Dickman, M. 2011. Genomic analysis of the necrotrophic fungal pathogens Sclerotinia sclerotiorum and Botrytis cinerea. PLoS Genet. 7:e1002230.

Atwell, S., Corwin, J. A., Soltis, N. E., Subedy, A., Denby, K. J., and Kliebenstein, D. J. 2015. Whole genome resequencing of Botrytis cinerea isolates identifies high levels of standing diversity. Front. Microbiol. 6:996.

Baraldi, E., Bertolini, P., Chierici, E., Trufelli, B., and Luiselli, D. 2002. Genetic diversity between Botrytis cinerea isolates from unstored and cold stored kiwi fruit. Phytopathologische Z. 150:629-635.

Barrett, R. D. H., and Schluter, D. 2008. Adaptation from standing genetic variation. Trends Ecol. Evol. Amst. 23:38-44.

Bradshaw, A. D. 1965. Evolutionary significance of phenotypic plasticity in plants. Adv. Genet. 13:115-155.

Calpas, J. T., Konschuh, M. N., Toews, C. C., and Tewari, J. P. 2006 Relationships among isolates of Botrytis cinerea collected from greenhouses and field locations in Alberta, based on RAPID analysis. Can. J. Plant Pathology 28:109-124.

Cao, H., Glazebrook, J., Clarke, J. D., Volko, S., and Dong, X. 1997. The Arabidopsis NPRl gene that controls systemic acquired resistance encodes a novel protein containing ankyrin repeats. Cell 88:57-63.

Denby, K. J., Kumar, P., and Kliebenstein, D. J. 2004. Identification of Botrytis cinerea susceptibility loci in Arabidopsis thaliana. Plant J. 38: 473-486.
Ferrari, S., Galletti, R., Denoux, C., De Lorenzo, G., Ausubel, F. M., and Dewdney, J. 2007. Resistance to Botrytis cinerea induced in Arabidopsis by elicitors is independent of salicylic acid, ethylene, or jasmonate signaling but requires PHYTOALEXIN DEFICIENT3. Plant Physiol. 144:367-379

Ferrari, S., Plotnikova, J.M., De Lorenzo, G., and Ausubel, F.M. 2003 Arabidopsis local resistance to Botrytis cinerea involves salicylic acid and camalexin and requires EDS4 and PAD2, but not SID2, EDS5 or PAD4. Plant J. 35:193-205.

Forsman, A. 2014. Rethinking phenotypic plasticity and its consequences for individuals, populations and species. Heredity 115:276-284.

Fournier, E., and Giraud, T. 2008. Sympatric genetic differentiation of a generalist pathogenic fungus, Botrytis cinerea, on two different host plants, grapevine and bramble. J. Evol. Biol. 21:122-132.

Fournier, E., Giraud, T., Loiseau, A., Vautrin, D., Estoup, A., Solignac, M., Cornuet, J. M., and Brygoo, Y. 2002. Characterization of nine polymorphic microsatellite loci in the fungus Botrytis cinerea (Ascomycota). Mol. Ecol. Notes 2:253-255.

Giraud, T., Fortini, D., Levis, C., Lamarque, C., Leroux, P., Lobuglio, K., and Brygoo, Y. 1999. Two sibling species of the Botrytis cinerea complex, transposa and vacuma, are found in sympatry on numerous host plants. Phytopathology 89:967-973.

Giraud, T., Fortini, D., Levis, C., Leroux, P., and Brygoo, Y. 1997. RFLP markers show genetic recombination in Botryotinia fuckeliana (Botrytis cinerea) and transposable elements reveal two sympatric species. Mol. Biol. Evol. 14:1177-1185.

Iacomi-Vasilescu, B., Bataille-Simoneau, N., Campion, C., Dongo, A., Laurent, E., Serandat, I., Hamon, B., and Simoneau, P. 2008. Effect of null mutations in the AbNIK1 gene on saprophytic and parasitic fitness of Alternaria brassicicola isolates highly resistant to dicarboximide fungicides. Plant Pathol. 57:937-947.

Kerwin, R., Feusier, J., Corwin, J., Rubin, M., Lin, C., Muok, A., Larson, B., Li, B., Joseph, B., Francisco, M., Copeland, D., Weinig, C., and Kliebenstein, D. J. 2015. Natural genetic variation in Arabidopsis thaliana defense metabolism genes modulates field fitness. eLife 4:4.

Kliebenstein, D. J., Gershenzon, J., and Mitchell-Olds, T. 2001a Comparative quantitative trait loci mapping of aliphatic, indolic and benzylic glucosinolate production in Arabidopsis thaliana leaves and seeds. Genetics 159:359-370.

Kliebenstein, D. J., Kroymann, J., Brown, P., Figuth, A., Pedersen, D., Gershenzon, J., and Mitchell-Olds, T. 2001b. Genetic control of natural variation in Arabidopsis glucosinolate accumulation. Plant Physiol. 126: 811-825.

Kliebenstein, D. J., Rowe, H. C., and Denby, K. J. 2005. Secondary metabolites influence Arabidopsis/Botrytis interactions: Variation in host production and pathogen sensitivity. Plant J. 44:25-36.

Kretschmer, M., Leroch, M., Mosbach, A., Walker, A.-S., Fillinger, S., Mernke, D., Schoonbeek, H.-J., Pradier, J.-M., Leroux, P., De Waard, M. A., and Hahn, M. 2009. Fungicide-driven evolution and molecular basis of multidrug resistance in field populations of the grey mould fungus Botrytis cinerea. PLoS Pathog. 5:e1000696.

Le, S., Josse, J., and Husson, F. 2008. FactoMineR: An R package for multivariate analysis. J. Stat. Softw. 25:1-18.

Ma, Z. H., and Michailides, T. J. 2005. Genetic structure of Botrytis cinerea populations from different host plants in California. Plant Dis 89:1083-1089.

McDonald, B. A., and Linde, C. 2002. Pathogen population genetics, evolutionary potential, and durable resistance. Annu. Rev. Phytopathol 40:349-379.

Munoz, G., Hinrichsen, P., Brygoo, Y., and Giraud, T. 2002. Genetic characterisation of Botrytis cinerea populations in Chile. Mycol. Res. 106 594-601.

Pigliucci, M., and Byrd, N. 1998. Genetics and evolution of phenotypic plasticity to nutrient stress in Arabidopsis: Drift, constraints or selection? Biol. J. Linn. Soc. 64:17-40.

Pigliucci, M., and Kolodynska, A. 2001. Phenotypic plasticity to light intensity in Arabidopsis thaliana: Invariance of reaction norms and phenotypic integration. Evol. Ecol. 16:27-47.

Pigliucci, M., and Schlichting, C. D. 1996. Reaction norms of Arabidopsis. IV. Relationships between plasticity and fitnessn. Heredity 76:427-436.

Pinheiro, J. C., and Chao, E. C. 2006. Efficient Laplacian and adaptive Gaussian quadrature algorithms for multilevel generalized linear mixed models. J. Comput. Graph. Stat. 15:58-81.

Price, T. D., Qvarnstrom, A., and Irwin, D. E. 2003. The role of phenotypic plasticity in driving genetic evolution. Proc. R. Soc. B Biol. Sci. 270 1433-1440. 
R Development Core Team. 2015. R: A Language and Environment for Statistical Computing.The R Foundation for Statistical Computing, Vienna. Published online.

Rate, D. N., Cuenca, J. V., Bowman, G. R., Guttman, D. S., and Greenberg, J. T. 1999. The gain-of-function Arabidopsis acd6 mutant reveals novel regulation and function of the salicylic acid signaling pathway in controlling cell death, defenses, and cell growth. Plant Cell 11:1695-1708.

Rowe, H. C., and Kliebenstein, D. J. 2007. Elevated genetic variation within virulence-associated Botrytis cinerea polygalacturonase loci. Mol. PlantMicrobe Interact 20:1126-1137.

Rowe, H. C., and Kliebenstein, D. J. 2008. Complex genetics control natural variation in Arabidopsis thaliana resistance to Botrytis cinerea. Genetics 180:2237-2250.

Rowe, H. C., Walley, J. W., Corwin, J., Chan, E. K.-F., Dehesh, K., and Kliebenstein, D. J. 2010. Deficiencies in jasmonate-mediated plant defense reveal quantitative variation in Botrytis cinerea pathogenesis. PLoS Pathog. 6:e1000861.

Schlichting, C. D. 1986. The evolution of phenotypic plasticity in plants. Annu. Rev. Ecol. Syst. 17:667-693.

Schumacher, J., Pradier, J. M., Simon, A., Traeger, S., Moraga, J., Collado, I. G., Viaud, M., and Tudzynski, B. 2012. Natural variation in the VELVET gene bcvel1 affects virulence and light-dependent differentiation in Botrytis cinerea. PLoS One 7:e47840.

Slepecky, R. A., and Starmer, W. T. 2009. Phenotypic plasticity in fungi: A review with observations on Aureobasidium pullulans. Mycologia 101:823-832.

Smoot, M. E., Ono, K., Ruscheinski, J., Wang, P.-L., and Ideker, T. 2011. Cytoscape 2.8: New features for data integration and network visualization. Bioinformatics 27:431-432.

Staats, M., van Baarlen, P., Schouten, A., van Kan, J. A. L., and Bakker, F. T. 2007. Positive selection in phytotoxic protein-encoding genes of Botrytis species. Fungal Genet. Biol. 44:52-63.

Staats, M., and van Kan, J. A. L. 2012. Genome update of Botrytis cinerea strains B05.10 and T4. Eukaryot. Cell 11:1413-1414.
Suzuki, R., and Shimodaira, H. 2006. Pvclust: An R package for assessing the uncertainty in hierarchical clustering. Bioinformatics 22:1540-1542.

Turelli, M., and Barton, N. H. 2004. Polygenic variation maintained by balancing selection: Pleiotropy, sex-dependent allelic effects and $\mathrm{G} \times \mathrm{E}$ interactions. Genetics 166:1053-1079.

van Wees, S. 2008. Phenotypic analysis of Arabidopsis mutants: Trypan blue stain for fungi, oomycetes, and dead plant cells. Cold Spring Harbor Press, Cold Spring Harbor, NY, U.S.A. Published online.

Whitman, D. W., and Agrawal, A. A. 2009. What is phenotypic plasticity and why is it important. Pages 1-63 in: Phenotypic Plasticity in Insects: Mechanisms and Consequences. D. W. Whitman and T. N. Ananthakrishnan, eds. CABI, Wallingford, U.K.

Williamson, B., Tudzynski, B., Tudzynski, P., and van Kan, J. A. L. 2007. Botrytis cinerea: The cause of grey mould disease. Mol. Plant Pathol. 8:561-580.

Windram, O., Madhou, P., McHattie, S., Hill, C., Hickman, R., Cooke, E., Jenkins, D. J., Penfold, C. A., Baxter, L., Breeze, E., Kiddle, S. J., Rhodes, J., Atwell, S., Kliebenstein, D. J., Kim, Y. S., Stegle, O., Borgwardt, K., Zhang, C., Tabrett, A., Legaie, R., Moore, J., Finkenstadt, B., Wild, D. L., Mead, A., Rand, D., Beynon, J., Ott, S., Buchanan-Wollaston, V., and Denby, K. J. 2012. Arabidopsis defense against Botrytis cinerea: Chronology and regulation deciphered by highresolution temporal transcriptomic analysis. Plant Cell 24:3530-3557.

Xie, D. X., Feys, B. F., James, S., Nieto-Rostro, M., and Turner, J. G. 1998. COI1: An Arabidopsis gene required for jasmonate-regulated defense and fertility. Science 280:1091-1094.

Zhang, L. P., Lin, G. Y., Nino-Liu, D., and Foolad, M. R. 2003. Mapping QTLs conferring early blight (Alternaria solani) resistance in a Lycopersicon esculentum $\times$ L. hirsutum cross by selective genotyping. Mol. Breed. 12:3-19.

Zhou, N., Tootle, T. L., and Glazebrook, J. 1999. Arabidopsis PAD3, a gene required for camalexin biosynthesis, encodes a putative cytochrome P450 monooxygenase. Plant Cell 11:2419-2428. 